\title{
The preparation of $N$-acylbenzotriazoles from aldehydes
}

\author{
Alan R. Katritzky, Anatoliy V. Vakulenko, and Ritu Jain \\ Center for Heterocyclic Compounds, Department of Chemistry, University of Florida, \\ Gainesville, Florida 32611- 7200 \\ E-mail: katritzky@chem.ufl.edu
}

\section{Dedicated to our good friend Branko Stanovnik on his $65^{\text {th }}$ birthday}

(received 27 Aug 03; accepted 23 Oct 03; published on the web 30 Oct 03)

\begin{abstract}
$\mathrm{N}$-Acyl- (3a-g) and $\mathrm{N}$-alkyl-benzotriazoles (10a-c) were prepared from the corresponding aldehydes or alkanes, respectively, using a slight excess of $N$-chlorobenzotriazole in the presence of AIBN (2,2'-azobisisobutyronitrile) as an initiator.
\end{abstract}

Keywords: $\mathrm{N}$-Chlorobenzotriazole, $\mathrm{N}$-acylbenzotriazole, $\mathrm{N}$-alkylbenzotriazole, AIBN

\section{Introduction}

$\mathrm{N}$-Acylbenzotriazoles are versatile $\mathrm{N}$ - and C- acylating agents ${ }^{1-3}$ for the preparation of primary, secondary and tertiary amides ${ }^{2}$ including formamides ${ }^{4}$ and oxamides, ${ }^{5}$ cinnamoyl hydrazides, ${ }^{6}$ enaminones $^{3}$ and for the conversion of ketone enolates into $\beta$-diketones. ${ }^{7}$

Literature syntheses of $N$-acylbenzotriazoles (i) react acid chlorides with 1(trimethylsilyl)benzotriazole, ${ }^{8}$ the sodium salt of benzotriazole, ${ }^{9}$ or benzotriazole ${ }^{10,11}$; (ii) react carboxylic acids with 1-(methanesulfonyl)benzotriazole; ${ }^{6}$ or (iii) employ palladium catalyzed carbonylation of diaryliodonium salts in the presence of benzotriazole. ${ }^{12}$

In cases where the aldehyde is more readily available than the corresponding carboxylic acid, it is convenient to carry out the conversion of aldehyde RCHO into an acylating agent RCOX. Indeed, a number of methods are available for the direct conversion of aldehydes to the corresponding acid halides: (i) by the treatment of aromatic aldehydes with $\mathrm{SO}_{2} \mathrm{Cl}_{2}$ in the presence of a phosphonium catalyst; ${ }^{13}$ (ii) use of $N$-chlorosuccinimide; ${ }^{14,15}$ (iii) carbon tetrahalides at high temperatures; ${ }^{16}$ (iv) vapor phase photochemical halogenation; ${ }^{17}$ and (v) iodobenzene dichloride. ${ }^{18}$

$\mathrm{N}$-Chlorobenzotriazole has been widely used as (i) a mild oxidant ${ }^{19,20}$ for the preparation of aldehydes, ketones, ${ }^{21}$ azo-compounds, ${ }^{21}$ sulphoxides, ${ }^{20}$ sulfonimidoyl chlorides, ${ }^{22}$ nitrile oxides; ${ }^{23}$ (ii) a chlorinating agent ${ }^{24,25}$ and (iii) for addition reactions to olefins ${ }^{26-28}$ and 
isonitriles. ${ }^{29}$ Rees and Storr showed that the reactions of $N$-chlorobenzotriazole as an oxidant are radical in nature. ${ }^{26}$

We now show that $N$-chlorobenzotriazole (2) conveniently converts aldehydes $1 \mathbf{1 a}-\mathbf{g}$ into the corresponding $\mathrm{N}$-acylbenzotriazoles 3a-g (Scheme 1). Alcohols 8a and $\mathbf{8 h}$ are also converted into acylbenzotriazoles (Scheme 4), while substituted toluenes give the corresponding $N$ alkylbenzotriazoles 10a-c. All these reactions take place in the presence of $\mathrm{AIBN}^{15}$ as a radical initiator.

\section{Results and Discussion}

Preparation of $N$-acylbenzotriazoles 3a-g. Refluxing aldehydes $\mathbf{1 a}-\mathbf{g}$ with $N$ chlorobenzotriazole (2) in benzene for $0.5-4 \mathrm{~h}$ in the presence of catalytic AIBN led to the $N$ acylbenzotriazole; yields in 53-83\% except for the furyl derivative 3g. (Scheme 1) This resembles the known reaction of aldehydes with $\mathrm{N}$-bromosuccinimide to give the corresponding acid bromides using catalytic amount of AIBN as a radical initiator. ${ }^{15}$ Results are shown in Table 1. Known products were identified by m.p. and direct comparison of the NMR spectra. Compound $3 \mathbf{e}$ is novel and its structure is supported by elemental analysis and its spectra.

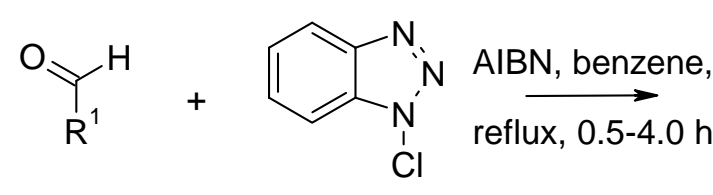

1 a-g
2<smiles>[R]C(=O)n1nnc2ccccc21</smiles>

3 a-g

\section{Scheme 1}

Table 1. Preparation of N-acylbenzotriazoles 3a-g

\begin{tabular}{cccccc}
\hline & $\mathrm{R}^{1}$ & $\begin{array}{c}\text { Time } \\
(\mathrm{h})\end{array}$ & Yield $(\%)$ & $\begin{array}{c}\text { m.p. } \\
\left({ }^{\circ} \mathrm{C}\right)\end{array}$ & $\begin{array}{c}\text { Lit. m.p. } \\
\left({ }^{\circ} \mathrm{C}\right)\end{array}$ \\
\hline 3a & $\mathrm{Ph}$ & 1.2 & 63 & $112-113$ & $112-113^{30-31}$ \\
3b & $\mathrm{C}_{2} \mathrm{H}_{5}$ & 2.5 & $53^{\mathrm{a}}$ & $77-78$ & $80-82^{31}$ \\
3c & $4-\mathrm{ClC}_{6} \mathrm{H}_{4}$ & 1.0 & 81 & $138-139$ & $138-139^{2}$ \\
3d & $\mathrm{C}_{6} \mathrm{H}_{5}\left(\mathrm{CH}_{2}\right)_{2}$ & 2.0 & 62 & $62-64$ & $63-64^{2}$ \\
3e & $3-$ thienyl & 3.0 & 56 & $118-120$ & Novel \\
3f & $\left(\mathrm{CH}_{3}\right)_{3} \mathrm{CCH}_{2}$ & 1.5 & 83 & $56-57$ & $56-57^{7}$ \\
3g & 2-furyl & 4.0 & 38 & $165-167$ & $165-167^{30}$ \\
\hline
\end{tabular}

aheating at $50-55^{\circ} \mathrm{C}$. 
Treatment of 4-methoxynaphthaldehyde (4) using our standard conditions formed a mixture of two compounds, however using 2 equivalents of $N$-chlorobenzotriazole gave 1H-1,2,3benzotriazol-1-yl-(3-chloro-4-methoxy-1-naphthyl)methanone (5). (Scheme 2) Boehlow et al. showed that ring halogenation takes place on the reaction of 4-methoxybenzaldehyde with $\mathrm{N}$ bromosuccinimide. ${ }^{32}$ Compound 5 was identified from its elemental analysis and spectral examination.<smiles></smiles>

\section{Scheme 2}

4-(Dimethylamino)-2-nitrobenzaldehyde (6), on treatment with 2 equiv of $N$ chlorobenzotriazole gave three ring halogenation products 7a-c (Scheme 3), which were separated by column chromatography. The structures of $\mathbf{7 a - c}$ were assigned on the basis of ${ }^{1} \mathrm{H}$ and ${ }^{13} \mathrm{C}$ NMR data and elemental analyses.

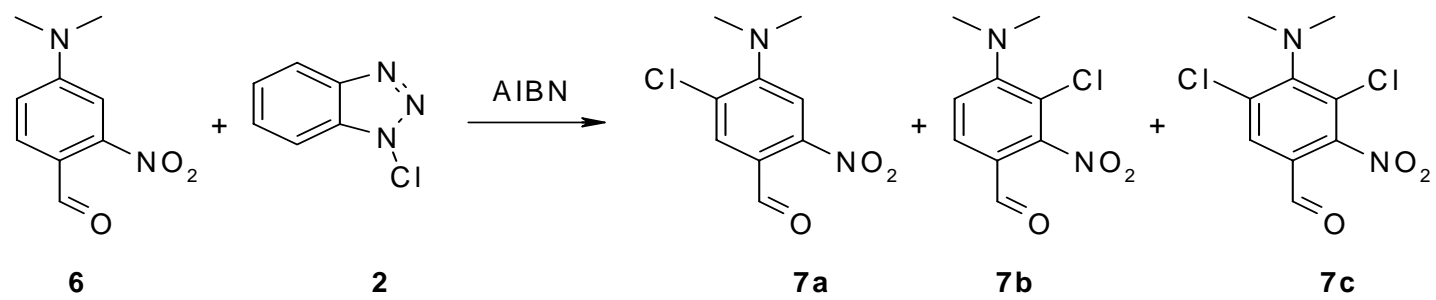

\section{Scheme 3}

The oxidation of alcohols into the corresponding aldehydes by $N$-chlorobenzotriazole has been reported previously. ${ }^{19}$ We now show that two equivalents of $N$-chlorobenzotriazole converted alcohols 8a,h as expected into the corresponding $N$-acylbenzotriazoles 3a,h (Scheme 4), which were purified by recrystallization. Known 3a was identical with the authentic sample, novel 3h was identified by elemental analysis and ${ }^{1} \mathrm{H}$ and ${ }^{13} \mathrm{C}$ NMR spectra. 


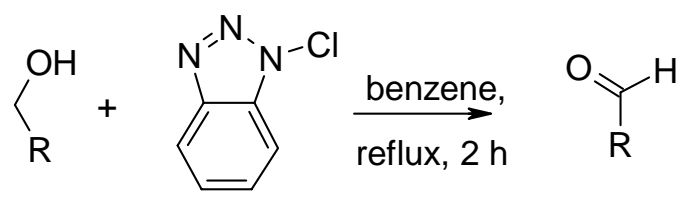

\begin{tabular}{c|ccc}
\multicolumn{2}{c}{$\mathbf{8 a , h}$} & \multicolumn{2}{c}{$\mathbf{2}$} \\
& $\mathrm{R}$ & $\begin{array}{c}\text { Time, } \\
(\mathrm{h})\end{array}$ & $\begin{array}{c}\text { Yield, } \\
(\%)\end{array}$ \\
\hline $\mathbf{3 a}$ & $\mathrm{Ph}$ & 1.0 & 60 \\
3h & $\mathrm{C}_{6} \mathrm{H}_{5} \mathrm{C}_{6} \mathrm{H}_{4}$ & 0.5 & 59
\end{tabular}

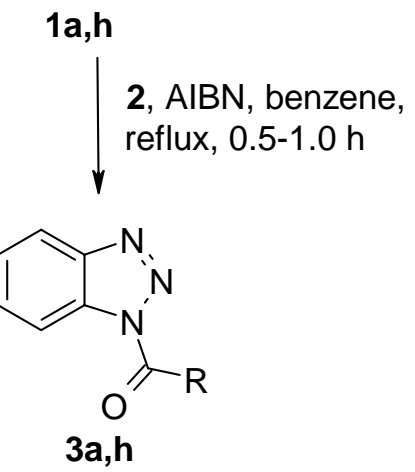

\section{Scheme 4}

Preparation of $\mathrm{N}$-alkylbenzotriazoles 10a-c. Reactions of $\mathrm{N}$-chlorobenzotriazole (2) with substituted toluenes 9a-c in the presence of AIBN at $90-100{ }^{\circ} \mathrm{C}$ for $21-48 \mathrm{~h}$ led to the formation of corresponding $N$-alkylbenzotriazoles $10 \mathrm{a}-\mathbf{c}$, similar to the radical bromination of the side chain of toluene previously observed by Slutter et al. ${ }^{33}$ on reaction with $N$-bromosuccinimide.

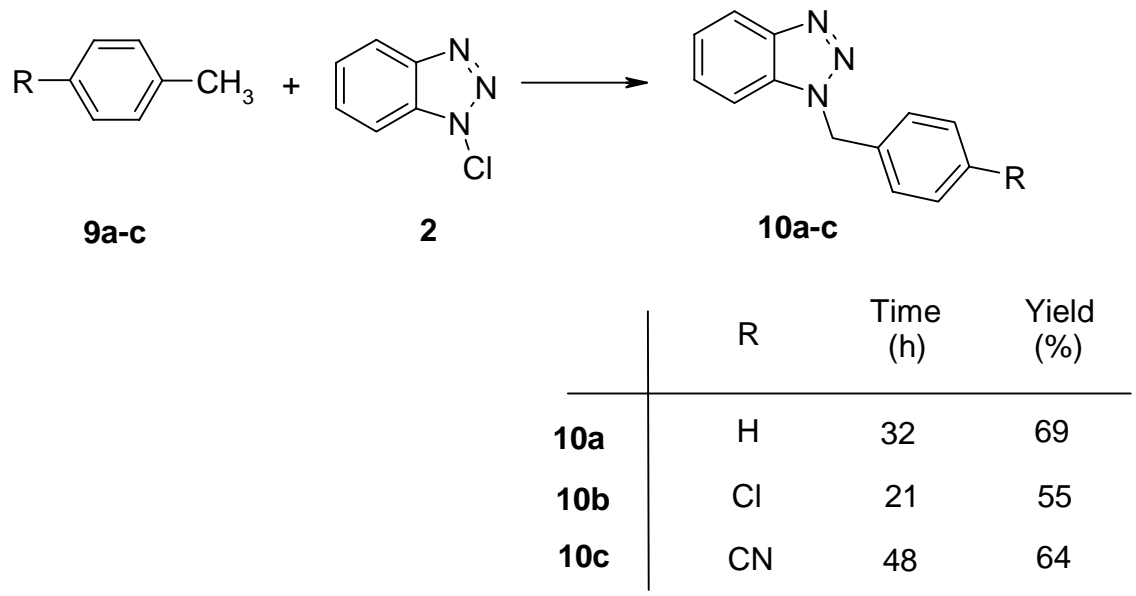

\section{Scheme 5}

Compounds 10a and 10b had singlets at $\delta 5.84$ and 5.82 identical with spectra previously published. ${ }^{34}$ Novel compound 10c was identified by elemental analysis and by its ${ }^{1} \mathrm{H}$ and ${ }^{13} \mathrm{C}$ NMR spectra.

$N$-Benzylbenzotriazoles are useful synthetically in many ways. Thus, lithiation with butyl lithium can be followed by the addition to aliphatic, aromatic, and $\alpha, \beta$-unsaturated aldehydes; ${ }^{35}$ 
cyclic and acyclic ketones; ${ }^{35,36}$ carboxylic esters; ${ }^{37}$ enamines ${ }^{38,39}$ and imides ${ }^{40}$ and leading to the formation of trans-alkenes, dienes , trienes and alkynes. 2-(Benzotriazol-1-yl)enamines (obtained from lithiated 1-(arylmethyl)benzotriazoles and nitriles) undergo rearrangement to give 2,2diarylquinazolines. ${ }^{41}$ 1-Benzylbenzotriazoles act as a 1,3-dipole synthons in Michael additioncyclization for the preparation of polysubstituted naphthalenes and phenanthrenes. ${ }^{42}$ Annulation of benzylbenzotriazole with 2-bromoacetaldehyde diethyl acetal followed by the treatment with BuLi and $N$-benzylideneaniline leads to the formation of 1,2,3-triarylpyrroles in one pot. ${ }^{43}$ [3+2] cycloaddition of benzyl benzotriazole with alkenes leads to the formation of functionalised indans. $^{44}$

Literature methods to prepare $N$-benzylbenzotriazoles include reactions of the corresponding alkane or arene with 1-chloromethylbenzotriazole in the presence of aluminium halide; ${ }^{45}$ or from the corresponding arylmethyl halide with benzotriazole. ${ }^{42,46}$

To summarize, we have developed one pot syntheses of $N$-acyl- and $N$-alkyl-benzotriazoles from the corresponding aldehydes/alcohols or toluenes respectively, utilizing $N$ chlorobenzotriazole in the presence of a radical initiator.

\section{Experimental Section}

General Procedures. Melting points were determined on a hot-stage apparatus and are uncorrected. NMR spectra were recorded in $\mathrm{CDCl}_{3}$ with TMS as the internal standard for ${ }^{1} \mathrm{H}$ (300 MHz) or a solvent as the internal standard for ${ }^{13} \mathrm{C}(75 \mathrm{MHz})$. Micro elemental analyses were performed on a Carlo Erba EA-1108 elemental analyzer.

Materials. 1-Chlorobenzotriazole (2) was synthesized according to the previously published procedure $^{47}$ as white crystals $\mathrm{mp} \quad 103-105{ }^{\circ} \mathrm{C} \quad\left(103-105{ }^{\circ} \mathrm{C}^{47}\right)$. 4-(Dimethylamino)-2nitrobenzaoldehyde (6) was synthesized according to the published procedure by Baumann et al. $^{48}$ and was confirmed by the NMR data and CHN analysis.

\section{General procedure for the synthesis of $N$-acylbenzotriazole from the corresponding aldehydes (3a-g)}

The mixture of corresponding aldehyde $(11 \mathrm{mmol}), N$-chlorobenzotriazole $(2.70 \mathrm{~g}, 17.6 \mathrm{mmol})$ and AIBN $(0.1 \mathrm{~g}, 0.6 \mathrm{mmol})$ in benzene $(80 \mathrm{~mL})$ were refluxed for the specified time (Scheme 1). To the cooled reaction mixture ethyl acetate was added, and the organic layer was washed with saturated aqueous $\mathrm{Na}_{2} \mathrm{CO}_{3}$, brine, and dried over $\mathrm{MgSO}_{4}$. The solvent was evaporated to give the crude product, which was purified by recrystallisation from the appropriate solvent to afford the pure compound.

1H-1,2,3-Benzotriazol-1-yl-(phenyl)methanone (3a). White needles from 2-propanol (63\%), mp $112-113{ }^{\circ} \mathrm{C}\left(112-113{ }^{\circ} \mathrm{C}^{30,31}\right)$; ${ }^{1} \mathrm{H}$ NMR $\delta 7.53-7.61(\mathrm{~m}, 3 \mathrm{H}), 7.68-7.74(\mathrm{~m}, 2 \mathrm{H}), 8.16-8.23$ $(\mathrm{m}, 3 \mathrm{H}), 8.40(\mathrm{~d}, J=8.2 \mathrm{~Hz}, 1 \mathrm{H}) ;{ }^{13} \mathrm{C}$ NMR $\delta 114.8,120.2,126.3,128.4,130.4,131.5,131.7$, $132.3,133.7,145.7,166.7$. 
1- (1H-1,2,3-Benzotriazol-1-yl)-1-propanone (3b). White prisms from 2-propanol (53 \%), $\mathrm{mp}$ $77-78{ }^{\circ} \mathrm{C}\left(80-82{ }^{\circ} \mathrm{C}^{31}\right) ;{ }^{1} \mathrm{H}$ NMR $\delta 1.43(\mathrm{t}, J=7.3 \mathrm{~Hz}, 3 \mathrm{H}), 3.47$ (q, $\left.J=7.4 \mathrm{~Hz}, 2 \mathrm{H}\right), 7.51(\mathrm{t}, J=$ $8.0 \mathrm{~Hz}, 1 \mathrm{H}), 7.66(\mathrm{t}, J=8.1 \mathrm{~Hz}, 1 \mathrm{H}), 8.12(\mathrm{~d}, J=8.2 \mathrm{~Hz}, 1 \mathrm{H}), 8.30(\mathrm{~d}, J=8.2 \mathrm{~Hz}, 1 \mathrm{H}) ;{ }^{13} \mathrm{C}$ NMR $\delta 8.3,29.1,114.4,120.1,126.0,130.3,131.1,146.1,173.3$. Anal. Calcd for $\mathrm{C}_{9} \mathrm{H}_{9} \mathrm{~N}_{3} \mathrm{O}: \mathrm{C}$, 61.70; H, 5.18; N, 23.99. Found: C, 61.77; H, 5.22; N, 24.12.

1H-1,2,3-Benzotriazol-1-yl-(4-chlorophenyl)methanone (3c). White needles from 2-propanol (81\%), mp 138-139 ${ }^{\circ} \mathrm{C}\left(138-139{ }^{\circ} \mathrm{C}^{2}\right) ;{ }^{1} \mathrm{H}$ NMR $\delta 7.54-7.59(\mathrm{~m}, 3 \mathrm{H}), 7.72(\mathrm{t}, J=8.1 \mathrm{~Hz}, 1 \mathrm{H})$, 8.16-8.22 (m, 3H), 8.38 (d, $J=8.2 \mathrm{~Hz}, 1 \mathrm{H}) ;{ }^{13} \mathrm{C}$ NMR $\delta 114.8,120.2,126.5,128.8,129.7$, $130.5,132.2,133.2,140.4,145.7,165.6$.

1-(1H-1,2,3-Benzotriazol-1-yl)-3-phenyl-1-propanone (3d). White prisms from benzene at 5$10{ }^{\circ} \mathrm{C}$ overnight $(62 \%), \operatorname{mp} 62-64{ }^{\circ} \mathrm{C}\left(63-64{ }^{\circ} \mathrm{C}^{2}\right) ;{ }^{1} \mathrm{H}$ NMR $\delta 3.23(\mathrm{t}, J=7.8 \mathrm{~Hz}, 2 \mathrm{H}), 3.77(\mathrm{t}$, $J=7.6 \mathrm{~Hz}, 2 \mathrm{H}), 7.19-7.32(\mathrm{~m}, 5 \mathrm{H}), 7.47-7.52(\mathrm{~m}, 1 \mathrm{H}), 7.61-7.67(\mathrm{~m}, 1 \mathrm{H}), 8.11(\mathrm{~d}, J=8.2 \mathrm{~Hz}$, $1 \mathrm{H}), 8.28(\mathrm{~d}, J=8.2 \mathrm{~Hz}, 1 \mathrm{H}) ;{ }^{13} \mathrm{C} \mathrm{NMR} \delta 30.1,37.1,114.4,120.1,126.1,126.5,128.4,128.6$, $130.3,131.0,139.8,146.1,171.6$.

1H-1,2,3-Benzotriazol-1-yl(3-thienyl)methanone (3e). Brownish microcrystals from 2propanol (56 \%), mp 118-120 ${ }^{\circ} \mathrm{C} ;{ }^{1} \mathrm{H}$ NMR (DMSO) $\delta 7.65(\mathrm{t}, J=7.7 \mathrm{~Hz}, 1 \mathrm{H}), 7.79-7.84(\mathrm{~m}$, $2 \mathrm{H}), 7.90(\mathrm{~d}, J=4.9 \mathrm{~Hz}, 1 \mathrm{H}), 8.28-8.34(\mathrm{~m}, 1 \mathrm{H}), 8.98($ br s, $1 \mathrm{H}) ;{ }^{13} \mathrm{C}$ NMR (DMSO) $\delta 114.4$, 120.0, 126.5, 127.3, 129.4, 130.7, 131.7, 132.5, 138.6, 145.0, 160.1. Anal. Calcd for $\mathrm{C}_{11} \mathrm{H}_{7} \mathrm{~N}_{3} \mathrm{OS}$ : C, 57.63; H, 3.08; N, 18.33. Found: C, 57.55; H, 2.92; N, 18.44.

1-(1H-1,2,3-Benzotriazol-1-yl)-3,3-dimethyl-1-butanone (3f). White prisms from hexanes (83\%), mp 56-57 ${ }^{\circ} \mathrm{C}\left(56-57{ }^{\circ} \mathrm{C}^{7}\right){ }^{1} \mathrm{H}$ NMR $\delta 1.17(\mathrm{~s}, 9 \mathrm{H}), 3.36(\mathrm{~s}, 2 \mathrm{H}), 7.48-7.54(\mathrm{~m}, 1 \mathrm{H}), 7.63-7.68$ $(\mathrm{m}, 1 \mathrm{H}), 8.12(\mathrm{~d}, J=8.4 \mathrm{~Hz}, 1 \mathrm{H}), 8.34(\mathrm{~d}, J=8.2 \mathrm{~Hz}, 1 \mathrm{H}) ;{ }^{13} \mathrm{C}$ NMR $\delta 29.8,32.0,47.1,114.6$, $120.1,126.0,130.2,131.1,146.2,171.3$.

1H-1,2,3-Benzotriazol-1-yl (2-furyl)methanone (3g). White microcrystals from 2-propanol (38 \%); mp $165-167{ }^{\circ} \mathrm{C}\left(165-167{ }^{\circ} \mathrm{C}^{30}\right) ;{ }^{1} \mathrm{H} \mathrm{NMR} \delta 6.74(\mathrm{dd}, J=3.7,1.6 \mathrm{~Hz}, 1 \mathrm{H}), 7.52-7.57(\mathrm{~m}$, $1 \mathrm{H}), 7.67-7.73(\mathrm{~m}, 1 \mathrm{H}), 7.88-7.88(\mathrm{~m}, 1 \mathrm{H}), 8.15-8.18(\mathrm{~m}, 2 \mathrm{H}), 8.42(\mathrm{~d}, J=8.4 \mathrm{~Hz}, 1 \mathrm{H}),{ }^{13} \mathrm{C}$ NMR $\delta 113.0,114.7,120.2,124.8,126.3,130.5,132.2,144.6,145.6,148.9,155.1$.

\section{Procedure for the synthesis of 1H-1,2,3-benzotriazol-1-yl (3-chloro-4-methoxy-1- naphthyl)methanone (5)}

The mixture of 4-methoxy-1-naphthaldehyde $(0.45 \mathrm{~g}, 2.4 \mathrm{mmol}), \mathrm{N}$-chlorobenzotriazole $(0.70 \mathrm{~g}$, $4.6 \mathrm{mmol})$ and AIBN $(0.1 \mathrm{~g}, 0.6 \mathrm{mmol})$ in benzene $(80 \mathrm{~mL})$ were refluxed for $4.0 \mathrm{~h}$, then stirred at room temperature overnight. To the reaction mixture, ethyl acetate was added $(50 \mathrm{ml})$, and the organic layer was washed with saturated aqueous $\mathrm{Na}_{2} \mathrm{CO}_{3}$, brine and dried over $\mathrm{MgSO}_{4}$. The solvent was evaporated to give the crude product, which was purified by column chromatography $\left(\mathrm{CH}_{2} \mathrm{Cl}_{2}\right.$ : Hexanes $\left.=1: 1\right)$ to afford the pure product. White microcrystals (57\%), mp 142-143 ${ }^{\circ} \mathrm{C} ;{ }^{1} \mathrm{H}$ NMR $\delta 4.14$ (s, 3H), 7.56-7.67 (m, 3H), 7.74-7.80 (m, 1H), 7.96 (s, $1 \mathrm{H}), 8.13-8.17(\mathrm{~m}, 1 \mathrm{H}), 8.19(\mathrm{~d}, J=8.2 \mathrm{~Hz}, 1 \mathrm{H}), 8.26-8.29(\mathrm{~m}, 1 \mathrm{H}), 8.47$ (d, J = 8.2 Hz, 1H); ${ }^{13} \mathrm{C}$ NMR $\delta 61.7,114.7,120.4,121.8,122.6,125.2,126.2,126.6,127.5,128.3,129.4,130.7$, 
131.4, 132.0, 132.4, 146.2, 155.7, 166.0. Anal. Calcd for $\mathrm{C}_{18} \mathrm{H}_{12} \mathrm{ClN}_{3} \mathrm{O}_{2}: \mathrm{C}, 64.01 ; \mathrm{H}, 3.58 ; \mathrm{N}$, 12.44. Found: C, 64.30; H, 3.46; N, 12.42 .

General procedure for the synthesis of substituted 4-(dimethylamino)-6-nitrobenzaldehyde (7a-c)

The mixture of 4-(dimethylamino)-2-nitrobenzaldehyde $\quad(0.97 \mathrm{~g}, \quad 5.0 \mathrm{mmol}), \quad N$ chlorobenzotriazole $(1.44 \mathrm{~g}, 9.4 \mathrm{mmol})$ and AIBN $(0.1 \mathrm{~g}, 0.6 \mathrm{mmol})$ in benzene $(80 \mathrm{~mL})$ were refluxed for $3.0 \mathrm{~h}$, then stirred at room temperature overnight. To the reaction mixture, ethyl acetate was added, and the organic layer was washed with saturated aqueous $\mathrm{Na}_{2} \mathrm{CO}_{3}$, brine, and dried over $\mathrm{MgSO}_{4}$. The solvent was evaporated to give the crude product, which was purified by column chromatography $\left(\mathrm{CH}_{2} \mathrm{Cl}_{2}\right.$ :Hexanes $\left.=6: 4\right)$ to give $\mathbf{7 a}-\mathbf{c}$.

5-Chloro-4-(dimethylamino)-2-nitrobenzaldehyde (7a). Orange microcrystals (17 \%), mp 92$94{ }^{\circ} \mathrm{C} ;{ }^{1} \mathrm{H}$ NMR $\delta 3.07$ (s, 6H), 7.52 (s, 1H), 7.95 (s, 1H), $10.25(\mathrm{~s}, 1 \mathrm{H}) ;{ }^{13} \mathrm{C}$ NMR $\delta$ 42.8, 113.7 , 122.5, 129.7, 132.4, 149.1, 154.1, 186.0. Anal. Calcd for $\mathrm{C}_{9} \mathrm{H}_{9} \mathrm{ClN}_{2} \mathrm{O}_{3}: \mathrm{C}, 47.28 ; \mathrm{H}, 3.97 ; \mathrm{N}$, 12.25. Found: C, 47.23; H, 3.82; N, 12.49 .

3-Chloro-4-(dimethylamino)-2-nitrobenzaldehyde (7b). Orange microcrystals (4\%), mp 95$97{ }^{\circ} \mathrm{C} ;{ }^{1} \mathrm{H}$ NMR $\delta 3.07$ (s, 6H), 7.09 (d, $\left.J=8.7 \mathrm{~Hz}, 1 \mathrm{H}\right), 7.73$ (d, J=8.7 Hz, 1H), 9.72 (s, 1H); ${ }^{13} \mathrm{C}$ NMR $\delta 43.0,116.0,118.6,119.8,129.9,130.3,155.7,184.8$. Anal. Calcd for $\mathrm{C}_{9} \mathrm{H}_{9} \mathrm{ClN}_{2} \mathrm{O}_{3}$ : C, 47.28; H, 3.97; N, 12.25. Found: C, 46.94; H, 3.77; N, 12.24.

3,5-Dichloro-4- (dimethylamino)-6-nitrobenzaldehyde (7c). Yellow needles (43\%), mp 63$64{ }^{\circ} \mathrm{C} ;{ }^{1} \mathrm{H}$ NMR $\delta 3.07$ (s, 6H), 7.83 (s, 1H), 9.73 (s, 1H); ${ }^{13} \mathrm{C}$ NMR $\delta$ 42.9, 100.2, 122.3, 124.3, 131.3, 134.0, 152.7, 183.9. Anal. Calcd for $\mathrm{C}_{9} \mathrm{H}_{8} \mathrm{Cl}_{2} \mathrm{~N}_{2} \mathrm{O}_{3}$ : C, 41.09; H, 3.07; N, 10.65. Found: C, $41.17 ; \mathrm{H}, 2.96 ; \mathrm{N}, 10.47$.

General procedure for the synthesis of $N$-acylbenzotriazole (3a, $3 h$ ) from the corresponding alcohols

The mixture of alcohol $(8 \mathrm{mmol})$ and $N$-chlorobenzotriazole $(1.78 \mathrm{~g}, 11.6 \mathrm{mmol})$ in benzene $(100 \mathrm{~mL})$ were refluxed for $2 \mathrm{~h}$, then AIBN $(0.1 \mathrm{~g}, 0.6 \mathrm{mmol})$ and the next portion of $N$ chlorobenzotriazole $(1.70 \mathrm{~g}, 11.1 \mathrm{mmol})$ were added. The solution was kept at $80{ }^{\circ} \mathrm{C}$ for the specified time (Scheme 1), then stirred at room temperature overnight. To the reaction mixture, ethyl acetate was added and the organic layer was washed with saturated aqueous $\mathrm{Na}_{2} \mathrm{CO}_{3}$, brine and dried over anhyd. $\mathrm{MgSO}_{4}$. The solvent was evaporated to give the crude product, which was purified by recrystallisation from the appropriate solvent to afford the pure compound.

1H-1,2,3-Benzotriazol-1-yl (phenyl)methanone (3a). White needles from 2-propanol (60\%), mp $112-113{ }^{\circ} \mathrm{C}\left(112-113{ }^{\circ} \mathrm{C}^{30}\right)$. The spectrum is identical with that for compound 3a prepared from benzaldehyde above.

1H-1,2,3-Benzotriazol-1-yl ([1,1'-biphenyl]-4-yl)methanone (3h). White prisms from 2propanol (59\%), mp 165-167 ${ }^{\circ} \mathrm{C} ;{ }^{1} \mathrm{H}$ NMR (DMSO) $\delta 7.45-7.58(\mathrm{~m}, 3 \mathrm{H}), 7.67$ (t, $J=7.7 \mathrm{~Hz}$, $1 \mathrm{H}), 7.82-7.88(\mathrm{~m}, 3 \mathrm{H}), 7.96(\mathrm{~d}, J=8.4 \mathrm{~Hz}, \mathrm{~A}$ part of AB system, 2H), $8.24(\mathrm{~d}, J=8.4 \mathrm{~Hz}, \mathrm{~B}$ part of $\mathrm{AB}$ system, $2 \mathrm{H}), 8.30-8.37(\mathrm{~m}, 2 \mathrm{H}) ;{ }^{13} \mathrm{C}$ NMR (DMSO) $\delta 114.5,120.0,126.5,126.6$, 
127.1, 128.6, 129.1, 130.1, 130.7, 131.8, 132.2, 138.7, 145.0, 145.2, 166.2. Anal. Calcd for $\mathrm{C}_{19} \mathrm{H}_{13} \mathrm{~N}_{3} \mathrm{O}$ : C, 76.24; H, 4.38; N, 14.04. Found: C, 75.83; H, 4.24; N, 14.18.

\section{General procedure for the synthesis of $\boldsymbol{N}$-alkyl- and $\boldsymbol{N}$-aryl- benzotriazoles (10a-c)}

The a mixture of $N$-chlorobenzotriazole $(2.30 \mathrm{~g}, 15.0 \mathrm{mmol})$ and AIBN $(0.1 \mathrm{~g}, 0.6 \mathrm{mmol})$ in excess of corresponding toluene as a reagent as well as solvent $(126.8 \mathrm{mmol})$ were heated at $105-110{ }^{\circ} \mathrm{C}$ for the specified time (Scheme 3). To the cooled reaction mixture, ethyl acetate was added, and the organic layer was washed with saturated aqueous $\mathrm{Na}_{2} \mathrm{CO}_{3}$, brine and dried over anhyd. $\mathrm{MgSO}_{4}$. The solvent was evaporated under reduced pressure to give the crude product, which was purified by recrystallization from the appropriate solvent to give the pure compound.

1-Benzyl-1H-1,2,3-benzotriazole (10a). White microcrystals form 2-propanol (69 \%), mp $115-116^{\circ} \mathrm{C}\left(115-116{ }^{\circ} \mathrm{C}^{34}\right) ;{ }^{1} \mathrm{H}$ NMR $\delta 5.84(\mathrm{~s}, 2 \mathrm{H}), 7.25-7.39(\mathrm{~m}, 8 \mathrm{H}), 8.06(\mathrm{~d}, J=7.83 \mathrm{~Hz}$, $1 \mathrm{H}) ;{ }^{13} \mathrm{C}$ NMR $\delta 52.2,109.7,120.0,123.8,127.3,127.5,128.4,128.9,132.7,134.7,146.3$.

1-(4-Chlorobenzyl)-1H-1,2,3-benzotriazole (10b). White microcrystals from 2-propanol (55\%), mp 98-99 ${ }^{\circ} \mathrm{C}\left(101-102{ }^{\circ} \mathrm{C}^{49}\right)$; ${ }^{1} \mathrm{H}$ NMR $\delta 5.82(\mathrm{~s}, 2 \mathrm{H}), 7.21(\mathrm{~d}, J=8.2 \mathrm{~Hz}, \mathrm{~A}$ part of the AB system, 2H), 7.29-7.46 (m, 5H), $8.08(\mathrm{~d}, J=8.8 \mathrm{~Hz}, 1 \mathrm{H}) ;{ }^{13} \mathrm{C} \mathrm{NMR} \delta 51.4,109.4,120.2,124.0$, 127.6, 128.9, 129.2, 132.6, 133.2, 134.4, 146.3. Anal. Calcd for $\mathrm{C}_{13} \mathrm{H}_{10} \mathrm{ClN}_{3}$ : C, 64.07; H, 4.14; N, 17.24. Found: C, 64.01; H, 3.95; N, 17.31.

4-(1H-1,2,3-Benzotriazol-1-ylmethyl)benzonitrile (10c). White prisms from 2-propanol (64 \%), mp 164- $166{ }^{\circ} \mathrm{C} ;{ }^{1} \mathrm{H}$ NMR $\delta 5.91(\mathrm{~s}, 2 \mathrm{H}), 7.33-7.49(\mathrm{~m}, 5 \mathrm{H}), 7.63(\mathrm{~d}, J=8.4 \mathrm{~Hz}$, A part of AB system, $2 \mathrm{H}), 8.10(\mathrm{~d}, J=8.2 \mathrm{~Hz}, 1 \mathrm{H}) ;{ }^{13} \mathrm{C} \mathrm{NMR} \delta 51.3,109.1,112.5,118.1,120.3,124.2$, 27.9, 128.0, 132.6, 132.7, 139.9, 146.2. Anal. Calcd for $\mathrm{C}_{14} \mathrm{H}_{10} \mathrm{~N}_{4}: \mathrm{C}, 71.78 ; \mathrm{H}, 4.30 ; \mathrm{N}, 23.92$. Found: C, 71.50; H, 4.30; N, 23.67.

\section{References}

1. Katritzky, A. R.; Yang, B.; Semenzin, D. J. J. Org. Chem. 1997, 62, 726.

2. Katritzky, A. R.; He, H.-Y.; Suzuki, K. J. Org. Chem. 2000, 65, 8210.

3. Katritzky, A. R.; Fang, Y.; Donkor, A.; Xu, J. Synthesis 2000, 2029.

4. Katritzky, A. R.; Chang, H.-X.; Yang, B. Synthesis 1995, 503.

5. Katritzky, A. R.; Levell, J. R.; Pleynet, D. P. M. Synthesis 1998, 153.

6. Katritzky, A. R.; Wang, M.; Zhang, S. ARKIVOC 2001, (ix), 19.

7. Katritzky, A. R.; Pastor, A. J. Org. Chem. 2000, 65, 3679.

8. Gasparini, J. P.; Gassend, R.; Maire, J. C.; Elguero, J. J. Organomet. Chem. 1980, 188, 141.

9. Baradarani, M. M.; Khalafy, J.; Prager, R. H. Aust. J. Chem. 1999, 52, 775.

10. Kreutzberger, A.; Dietz, E. Tetrahedron Lett. 1970, 1457.

11. Kreutzberger, A.; van der Goot, H. J. Heterocycl. Chem. 1975, 12, 665.

12. Wang, L.; Chen, Z.-C. Synth. Commun. 2001, 31, 1633.

13. Anon. Res. Discl. 1982, 219, 239; Chem. Abstr. 1982, 97, 144508x. 
14. Hebbelynck, M. F.; Martin, R. H. Bull. Soc. Chim. Belg. 1951, 60, 54.

15. Markó, I. E.; Mekhalfia, A. Tetrahedron Lett. 1990, 31, 7237.

16. Ansell, M. F. In The Chemistry of Acyl Halides; Patai, S., Ed.; Interscience Publishers: New York, 1972, p 55.

17. Buckley, E.; Whittle, E. Can. J. Chem. 1962, 40, 1611.

18. Mohandas, T. P.; Mamman, A. S.; Nair, P. M. Tetrahedron 1983, 39, 1187.

19. Rees, C. W.; Storr, R. C. J. Chem. Soc. (C) 1969, 1474.

20. Kingsbury, W. D.; Johnson, C. R. J. Chem. Soc., Chem. Comm. 1969, 365.

21. Rees, C. W.; Storr, R. C. J. Chem. Soc., Chem. Comm. 1968, 1305.

22. Johnson, C. R.; Jonsson, E. U.; Bacon, C. C. J. Org. Chem. 1979, 44, 2055.

23. Kim, J. N.; Ryu, E. K. Synth. Commun. 1990, 20, 1373.

24. Bowyer, P. M.; Iles, D. H.; Ledwith, A. J. Chem. Soc. (C) 1971, 16, 2775.

25. Cinquini, M.; Colonna, S. Synthesis 1972, 259.

26. Rees, C. W.; Storr, R. C. J. Chem. Soc. (C) 1969, 11, 1478.

27. Wender, P. A.; Cooper, C. B. Tetrahedron 1986, 42, 2985.

28. Katritzky, A. R.; Ignatchenko, A. V.; Lan, X.; Lang, H.; Stevens, C. V. Tetrahedron 1994, 50, 6005.

29. Katritzky, A. R.; Rogovoy, B.; Klein, C.; Insuasty, H.; Vvedensky, V.; Insuasty, B. J. Org. Chem. 2001, 66, 2854.

30. Katritzky, A. R.; Pastor, A.; Voronkov, M. V. J. Heterocycl. Chem. 1999, 36, 777.

31. Katritzky, A. R.; Shobana, N.; Pernak, J.; Afridi, A. S.; Fan, W.-Q. Tetrahedron 1992, 48, 7817.

32. Boehlow, T. R.; Harburn, J. J.; Spilling, C. D. J. Org. Chem. 2001, 66, 3111.

33. Sutter, M. Tetrahedron Lett. 1989, 30, 5417.

34. Katritzky, A. R.; Oniciu, D. C.; Ghiviriga, I. Synth. Commun. 1997, 27, 1613.

35. Katritzky, A. R.; Cheng, D.; Henderson, S. A.; Li, J. J. Org. Chem. 1998, 63, 6704.

36. Katritzky, A. R.; Li, J. J. Org. Chem. 1997, 62, 238.

37. Katritzky, A. R.; Cheng, D.; Li, J. J. Org. Chem. 1998, 63, 3438.

38. Katritzky, A. R.; Tymoshenko, D. O.; Belyakov, S. A. J. Org. Chem. 1999, 64, 3332.

39. Paventi, M.; Elce, E.; Jackman, R. J.; Hay, A. S. Tetrahedron Lett. 1992, 33, 6405.

40. Katritzky, A. R.; Gordeev, M. F. J. Chem. Soc., Perkin Trans. 1 1992, 1295.

41. Katritzky, A. R.; Yang, B.; Jiang, J. J. Org. Chem. 1995, 60, 246.

42. Katritzky, A. R.; Zhang, G.; Xie, L. J. Org. Chem. 1997, 62, 721.

43. Katritzky, A. R.; Wang, Z.; Li, J.; Levell, J. R. J. Heterocycl. Chem. 1997, 34, 1379.

44. Katritzky, A. R.; Zhang, G.; Xie, L. Synth. Commun. 1997, 27, 2467.

45. Katritzky, A. R.; Gordeev, M. F.; Greenhill, J. V.; Steel, P. J. J. Chem. Soc., Perkin Trans. 1 1992, 1111.

46. Katritzky, A. R.; Toader, D. J. Am. Chem. Soc. 1997, 119, 9321.

47. Hughes, T. V.; Hammond, S. D.; Cava, M. P. J. Org. Chem. 1988, 63, 401.

48. Bauman, H.; Grychtol, K.; Oberlinner, A. Ger. Offen. 2363458 1975; Chem. Abstr. 1976, 84, 4662z.

49. Katritzky, A. R.; Toader, D.; Xie, L. J. Org. Chem. 1996, 61, 7571. 